\title{
Experimental and Numerical Studies of One-Dimensional and Three-Dimensional Chaotic Open Systems
}

\author{
M. Ławniczak, O. Hul, Sz. Bauch and L. Sirko \\ Institute of Physics, Polish Academy of Sciences, al. Lotników 32/46, 02-668 Warszawa, Poland

\begin{abstract}
We present the results of experimental studies of microwave irregular networks and a three-dimensional microwave rough cavity in the presence of absorption. Microwave networks are also analyzed numerically. Microwave networks simulate one-dimensional quantum graphs. The networks consist of coaxial cables connected by joints and attenuators to control absorption. Three-dimensional microwave rough cavities have no formal analog in quantum 3D systems. However, some statistical properties of their spectra such as the level spacing distribution confirms that they belong to the wave-chaotic systems. Absorption of the cavity was changed by using a foam microwave absorber.
\end{abstract}

PACS numbers: 05.45.Mt, 03.65.Nk

\section{Introduction}

Quantum graphs of connected one-dimensional wires were introduced by Pauling more than seventy years ago [1]. Quantum graphs are often considered as idealizations of physical networks in the limit where the lengths of the wires greatly exceed their widths assuming that propagating waves remain in a single transverse mode. Among the systems modeled by quantum graphs are, e.g., electromagnetic optical waveguides [2, 3], mesoscopic systems $[4,5]$, quantum wires $[6,7]$ and excitation of fractons in fractal structures $[8,9]$. Recent works have shown that quantum graphs provide an excellent system for studying quantum chaos [10-19] and localization phenomena [20-22]. Quantum graphs with absorption, more realistic but more complicated systems, have been recently studied in $[16,17,23-25]$. The distribution of the reflection coefficient $P(R)$ and the distributions of the Wigner reaction matrix [26] (in the literature often called $K$ matrix [27]) for networks with time reversal symmetry (TRS) in the presence of moderate absorption strength $\gamma=2 \pi \Gamma / \Delta \leq 7.7$, where $\Gamma$ is the absorption width and $\Delta$ is the mean level spacing, have been investigated in $[17,28]$. Quite recently, the distribution of the reflection coefficient $P(R)$ and the distributions of the Wigner reaction matrix for networks with TRS in the presence of much stronger absorption $(\gamma>19)$ have been studied experimentally and numerically in [25].

Due to experimental problems there are few experimental studies devoted to three-dimensional (3D) chaotic microwave cavities [29-34]. In a seminal experiment Deus et al. [29] have measured eigenfrequencies of the 3D chaotic (irregular) microwave cavity in order to confirm that their distribution displays behavior characteristic of classically chaotic quantum systems, viz., the Wigner distribution. In other experiments [30-32] the periodic orbits, the distributions of the frequency shifts caused by the external perturbation and a trace formula for chaotic 3D cavities have been respectively studied. Properties of nodal domains in a chaotic three-dimensional microwave rough billiard with the translational symmetry were studied in the series of papers [33, 34].

Three-dimensional chaotic cavities as well as properties of random electromagnetic vector field have been also scarcely studied theoretically [35-37]. No experiments with chaotic 3D microwave cavities in the presence of strong absorption have been reported so far.

In this paper we present the results of the experimental and numerical studies of the distribution $P(R)$ of the reflection coefficient $R$ and the distributions of the Wigner reaction matrix [26] for microwave networks that correspond to graphs with time reversal symmetry $(\beta=1$ symmetry class of random matrix theory [38]) in the presence of absorption. Our experimental results are compared with the exact theoretical predictions of Savin et al. [39]. We also present the results of the experimental study of the distribution $P(R)$ of the reflection coefficient $R$ for the 3D chaotic microwave cavity in the presence of strong absorption.

In the case of systems coupled by a single-channel antenna, the $K$ matrix and the scattering matrix $S$ are related by

$$
S=\frac{1-\mathrm{i} K}{1+\mathrm{i} K} .
$$

The function $K=-\mathrm{i} Z$ and therefore the scattering matrix $S=(1-Z) /(1+Z)$ are directly connected with the impedance $Z$, which has been recently measured in a microwave cavity experiment [40,41]. The $S$ matrix can be parameterized as

$$
S=\sqrt{R} \mathrm{e}^{\mathrm{i} \theta},
$$

where $R$ is the reflection coefficient and $\theta$ is the phase.

After pioneering work of López et al. [42] there came theoretical studies of the properties of statistical distributions of the $S$ matrix with direct processes and imperfect coupling [43-45]. In a recent experiment work [46] the distribution of the $S$ matrix for chaotic microwave 
cavities with absorption was investigated. The distribution $P(R)$ of the reflection coefficient $R$ in Eq. (2), at the beginning investigated theoretically in the strong absorption limit [47], has been recently known for any dimensionless absorption strength $\gamma[39]$. For systems with TRS $(\beta=1)$ Méndez-Sánchez et al. [48] studied $P(R)$ experimentally, and Savin et al. [39] found an exact formula for $P(R)$. Beenakker and Brouwer [49] calculated $P(R)$ for systems violating time reversal symmetry $(\beta=2)$, in the case of a perfectly coupled, single-channel lead.

For systems with TRS, the explicit analytic expression for the distribution $P(R)$ of the reflection coefficient $R$ is given by [39]:

$$
P(R)=\frac{2}{(1-R)^{2}} P_{0}\left(\frac{1+R}{1-R}\right) .
$$

The probability distribution $P_{0}(x)$ is given by the expression

$$
P_{0}(x)=-\frac{\mathrm{d} W(x)}{\mathrm{d} x},
$$

where the integrated probability distribution $W(x)$ is expressed by the formula [39]:

$$
\begin{gathered}
W(x)=\frac{x+1}{4 \pi}\left[f_{1}(w) g_{2}(w)+f_{2}(w) g_{1}(w)\right. \\
\left.+h_{1}(w) j_{2}(w)+h_{2}(w) j_{1}(w)\right]_{w=(x-1) / 2} .
\end{gathered}
$$

The functions $f_{1}, g_{1}, h_{1}, j_{1}$ are defined as follows:

$$
\begin{aligned}
& f_{1}(w)=\int_{w}^{\infty} \mathrm{d} t \frac{\sqrt{t|t-w|} \mathrm{e}^{-\gamma t / 2}}{(1+t)^{3 / 2}} \\
& \times\left(1-\mathrm{e}^{-\gamma}+\frac{1}{t}\right), \\
& g_{1}(w)=\int_{w}^{\infty} \mathrm{d} t \frac{\mathrm{e}^{-\gamma t / 2}}{\sqrt{t|t-w|}(1+t)^{3 / 2}}, \\
& h_{1}(w)=\int_{w}^{\infty} \mathrm{d} t \frac{\sqrt{|t-w|} \mathrm{e}^{-\gamma t / 2}}{\sqrt{t(1+t)}} \\
& \times\left[\gamma+\left(^{-\gamma}\right)(\gamma t-2)\right] \\
& j_{1}(w)=\int_{w}^{\infty} \mathrm{d} t \frac{\mathrm{e}^{-\gamma t / 2}}{\sqrt{t|t-w|}(1+t)^{1 / 2}} .
\end{aligned}
$$

Their counterparts with the index 2 are given by the same expressions but the integration is performed in the interval $t \in[0, w]$ instead of $[w, \infty)$.

The distributions of the imaginary and the real parts $P(v)$ and $P(u)$ of the $K$ matrix [27] can be also expressed by the probability distribution $P_{0}(x)$ :

$$
P(v)=\frac{\sqrt{2}}{\pi v^{3 / 2}} \int_{0}^{\infty} \mathrm{d} q P_{0}\left[q^{2}+\frac{1}{2}\left(v+\frac{1}{v}\right)\right],
$$

and

$$
\begin{gathered}
P(u)=\frac{1}{2 \pi \sqrt{u^{2}+1}} \int_{0}^{\infty} \mathrm{d} q P_{0} \\
\times\left[\frac{\sqrt{u^{2}+1}}{2}\left(q+\frac{1}{q}\right)\right],
\end{gathered}
$$

where $-v=\operatorname{Im} K<0$ and $u=\operatorname{Re} K$ are, respectively, the imaginary and real parts of the $K$ matrix.

\section{Experimental investigation of microwave networks}

Experimentally the hexagonal microwave networks (graphs) were investigated. The analogy between microwave networks and quantum graphs is based upon the equivalency of the telegraph equation describing microwave circuits and of the Schrödinger equation describing quantum systems [16]. One should point out here that there are also other systems often used in studying wave chaos such as microwave flat cavities [50], ray-splitting cavities [51-54] and atoms interacting with strong microwave fields [55-57]. Similarly to the graphs the flat microwave cavities simulate quantum billiards due to the equivalence between the Schrödinger equation and the Helmholtz equation.

A microwave network is built of coaxial cables (bonds), which are connected by joints (vertices). A coaxial cable consists of an inner conductor of radius $r_{1}$ surrounded by a concentric conductor of inner radius $r_{2}$. The space between the inner and the outer conductors is filled with a homogeneous material having a dielectric constant $\varepsilon$. Microwave graphs simulate quantum systems up to the cut-off frequency $\nu_{\mathrm{c}} \simeq \frac{c}{\pi\left(r_{1}+r_{2}\right) \sqrt{\varepsilon}}$ below, which only the fundamental TEM mode (Lecher wave) can propagate inside the coaxial cable. For SMA-RG-402 (semi-rigid) and Sucoform 141 (handformable) cables, which we use in the experiments, $r_{1}=0.05 \mathrm{~cm}, r_{2}=0.15 \mathrm{~cm}$ and $\varepsilon \approx 2.08$, single mode propagation occurs below $32.9 \mathrm{GHz}$. In our experiments we employ only fully connected networks (each vertex of the network is connected with each others) without any multiple bonds and loops. The number of undirected bonds for such networks is $B=N(N-1) / 2$, where $N$ is the number of vertices. For the vertices we used five five-arm joints and one six-arm joint which connected a network to the microwave network analyzer. To avoid the degeneracy of the observed spectrum we choose the lengths of the bonds to be rationally independent (irregular microwave graphs).

Primary absorption in microwave networks is due to losses in SMA cables. This relatively weak absorption $A(f)$ strongly depends on the microwave frequency $f$ : $A(f)=a \sqrt{f}+b f$. In the above formula the frequency $f$ is expressed in $\mathrm{GHz}$, and $a=0.3631 \mathrm{~dB} /\left(\mathrm{m} \mathrm{GHz}^{1 / 2}\right)$ and $b=0.0377 \mathrm{~dB} /(\mathrm{m} \mathrm{GHz})$ [58]. Stronger and more controllable absorption in the networks can be introduced by using microwave attenuators whose absorption in a wide frequency range, $0-14 \mathrm{GHz}$ in our case, almost does not depend on frequency. In numerical calculations weak absorption inside the cables can be described with the help of complex wave vector [16] while strong absorption inside attenuators can be described by a simple optical potential [25, 59].

Equation (1) holds for systems with absorption but without direct processes. The case of imperfect coupling and direct processes present $\left|\left\langle S_{\mathrm{s}}\right\rangle\right|>0$, where $S_{\mathrm{s}}$ is the scattering matrix of the system, can be mapped onto that of perfect one using two different approaches: the impedance approach and the parametrization one. 
The impedance approach was used to find the distribution $P(R)$ of the reflection coefficient $R$ and the distributions of imaginary and real parts of Wigner's reaction matrix $K$ for microwave networks with absorption $[40,41]$.

In this approach one can obtain the real and imaginary parts of the normalized impedance

$$
Z=\frac{\operatorname{Re} Z_{\mathrm{s}}+\mathrm{i}\left(\operatorname{Im} Z_{\mathrm{s}}-\operatorname{Im} Z_{\mathrm{r}}\right)}{\operatorname{Re} Z_{\mathrm{r}}}
$$

of a chaotic microwave system, where $Z_{\mathrm{s}(\mathrm{r})}=Z_{0}(1+$ $\left.S_{\mathrm{s}(\mathrm{r})}\right) /\left(1-S_{\mathrm{s}(\mathrm{r})}\right)$ is the system (radiation) impedance expressed by the system (radiation) scattering matrix $S_{\mathrm{s}(\mathrm{r})}$ and $Z_{0}$ is the characteristic impedance of the transmission line. The radiation impedance $Z_{\mathrm{r}}$ is the impedance seen at the output of the coupling structure for the same coupling geometry, but with the vertices (walls) of the system removed to infinity. Wigner's reaction matrix $K$ can be expressed by the normalized impedance $K=-\mathrm{i} Z$ and the scattering matrix $S$ of a network for the perfect coupling case (no direct processes present) can be extracted from the formula $S=(1-Z) /(1+Z)$.

The parameterization approach was applied to find the distribution $P(R)$ of the reflection coefficient $R$ of the 3D chaotic cavity with absorption. In this case an imperfect coupling $\left|\left\langle S_{\mathrm{s}}\right\rangle\right|>0$ with the cavity and direct processes were eliminated using the following mapping [27]:

$$
S=\frac{S_{\mathrm{s}}-\left|\left\langle S_{\mathrm{s}}\right\rangle\right|}{1-\left|\left\langle S_{\mathrm{s}}\right\rangle\right| S_{\mathrm{s}}}
$$

where $S_{\mathrm{s}}$ is the scattering matrix of the cavity measured using a single-channel antenna (imperfect coupling and direct processes might be present) while $S$ is the scattering matrix of the cavity for the perfect-coupling case (no direct processes present).

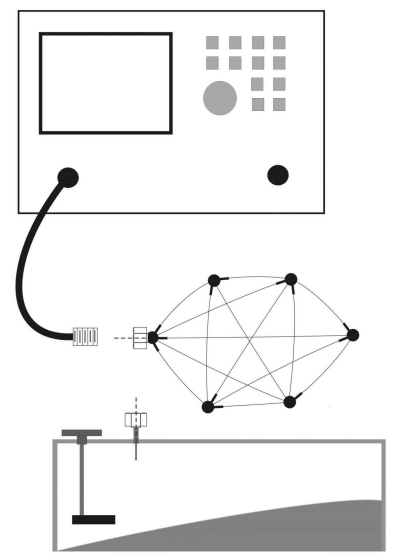

Fig. 1. The scheme of the experimental setup for measurements of the scattering matrix $S_{\mathrm{s}}$ of the microwave networks and the 3D microwave cavity. In the measurements Agilent E8364B microwave vector network analyzer was used. The graphs and the cavity were connected to the analyzer through the lead HP 85131-60016 flexible microwave cable.
We used Agilent E8364B microwave vector network analyzer to measure the scattering matrix $S_{\mathrm{s}}$ of the systems. The graphs and the cavity were connected to the analyzer through the lead HP 85131-60016 flexible microwave cable (see Fig. 1).

Typically, measurements of the scattering matrix $S_{\mathrm{s}}$ of 30 to 90 different configurations of the microwave graphs were averaged to obtain the distributions of $P(R)$, and $P(v), P(u)$, imaginary and real parts of $K$ matrix, for a given absorption. The sets of attenuators of the values 1 and $2 \mathrm{~dB}$ were used to realize graphs of given absorption. The different configurations were realized by exchanging one or two bonds of the graph into bonds of other length. However, the changes of the graphs length were kept small enough, less than $14 \%$ of the total length, to avoid their substantial influence on the absorption. The average optical length of the graphs was $5.70 \mathrm{~m}$. The measurements were performed in the frequency range $0-14 \mathrm{GHz}$.

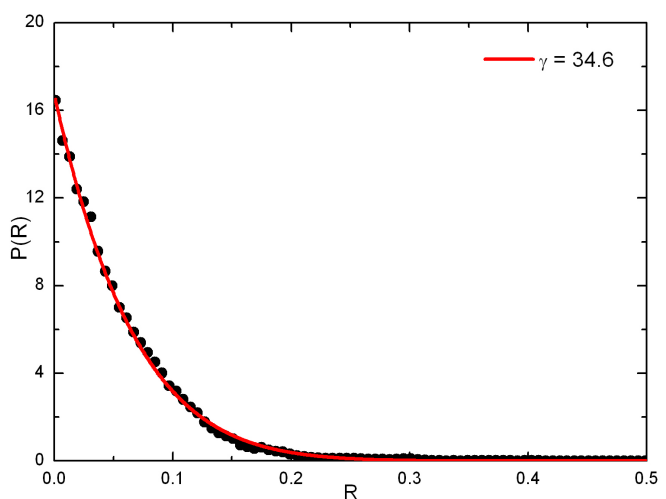

Fig. 2. The experimental distribution $P(R)$ of the reflection coefficient $R$ for the microwave fully connected hexagon networks for $\bar{\gamma}=34.6$ (solid circles). The corresponding theoretical distribution $P(R)$ is marked by the solid line.

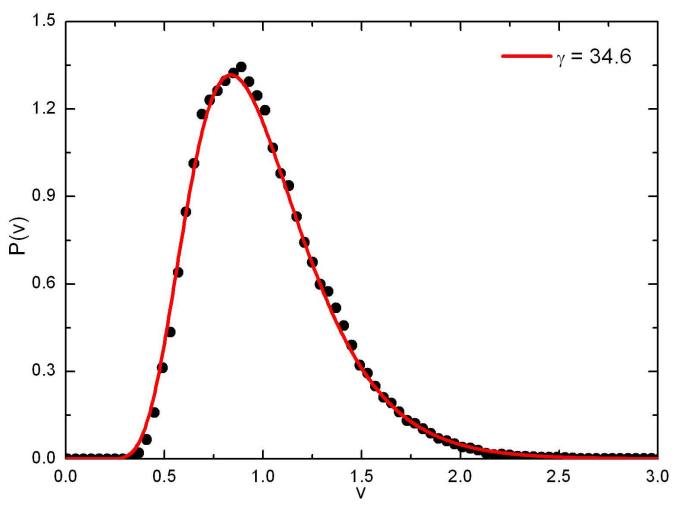

Fig. 3. The experimental distribution $P(v)$ of the imaginary part of the $K$ matrix for the mean absorption parameter $\bar{\gamma}=34.6$ (solid circles). The corresponding theoretical distribution $P(v)$ is marked by the solid line. 


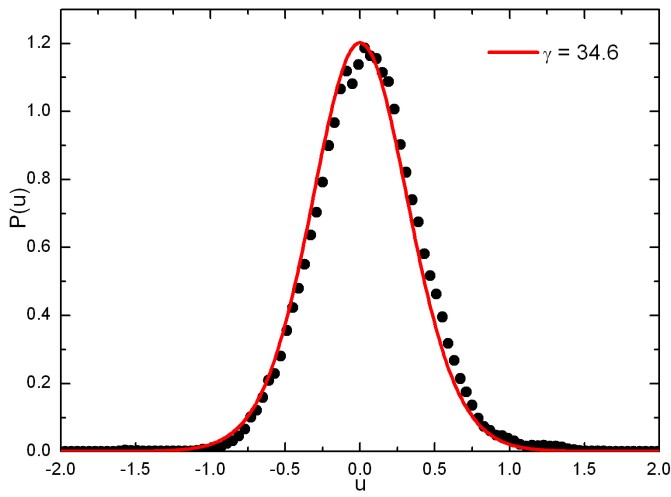

Fig. 4. The experimental distribution $P(u)$ of the real part of the $K$ matrix for the mean absorption parameter $\bar{\gamma}=34.6$ (solid circles). The corresponding theoretical distribution $P(R)$ is marked by the solid line.

Figure 2 shows the distribution of the $P(R)$ for $\bar{\gamma}=$ 34.6 obtained by averaging the measurements of $S_{\mathrm{s}}$ of 19 configurations of the networks with nine $1 \mathrm{~dB}$ and six $2 \mathrm{~dB}$ attenuators and 22 networks with $2 \mathrm{~dB}$ attenuators only, with value of $\gamma$ in the range $27-43$. The very good agreement with the exact theoretical predictions of Savin et al. [39] is observed. Only a small excess in the vicinity of 0.025 and 0.075 of $R$ is seen. In Figs. 3 and 4 we present the distributions of the imaginary $P(v)$ and real $P(u)$ part of the $K$ matrix determined from the same measurements, also compared with the exact theoretical predictions of [39]. The $P(u)$ exhibits clear deviation, shift to right, from the theoretical curve, whereas only a trace of such behavior may be ascribed to the $P(v)$.

\section{Numerical investigation of microwave networks}

The experimentally measured fully connected hexagon networks were described in numerical calculations by quantum fully connected hexagon graphs with one lead attached to the six-arm vertex. In the numerical calculations of the scattering matrices $S_{\mathrm{s}}$ of the graphs, attenuators (absorption) were modelled by optical potentials $[25,59]$. Furthermore, the weak absorption inside the microwave cables was taken into account by replacing the real wave vector $k$ by the complex vector $k+\mathrm{i} a \sqrt{k}$ [60], where the absorption coefficient was assumed to be $a=0.009 \mathrm{~m}^{-1 / 2}[16,25]$.

In Figs. 5, 6 and 7 we show the distributions $P(R)$, $P(v)$ and $P(u)$ obtained by averaging of the results of numerical calculations for 202 configurations of the quantum graphs. The absorption modelled by optical potentials was equivalent to placing $1.5 \mathrm{~dB}$ attenuator at each bond of the graph and resulted in the same $\bar{\gamma}=34.6$ as in experimental results. The different configurations of graphs were obtained by changing the length of one or two bonds, keeping their total length almost constant. The remarkable agreement of all presented distributions

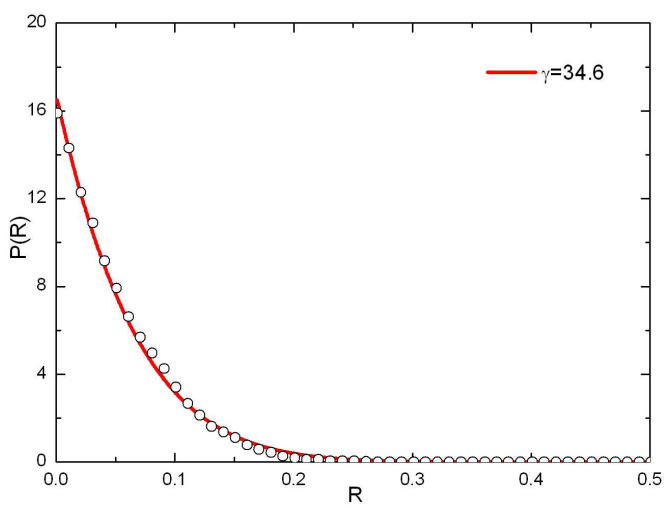

Fig. 5. The numerical distribution $P(R)$ of the reflection coefficient $R$ for fully connected hexagon quantum graphs for $\bar{\gamma}=34.6$ (empty circles). The corresponding theoretical distribution $P(R)$ is marked by the solid line.

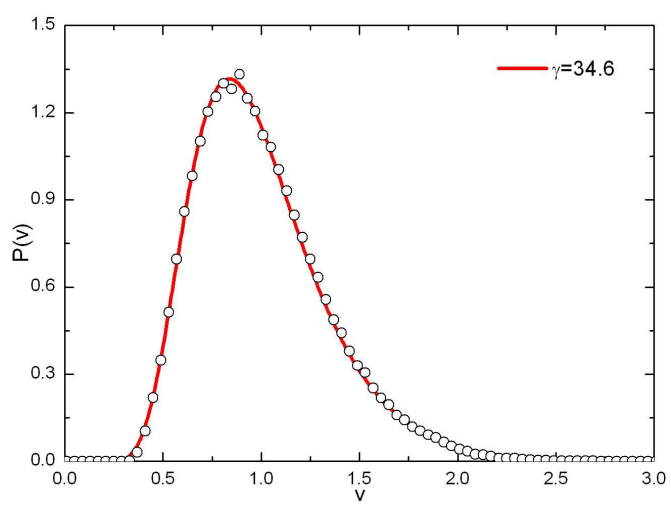

Fig. 6. The numerical distribution $P(v)$ of the imaginary part of the $K$ matrix for the mean absorption parameter $\bar{\gamma}=34.6$ (empty circles). The corresponding theoretical distribution $P(v)$ is marked by the solid line.

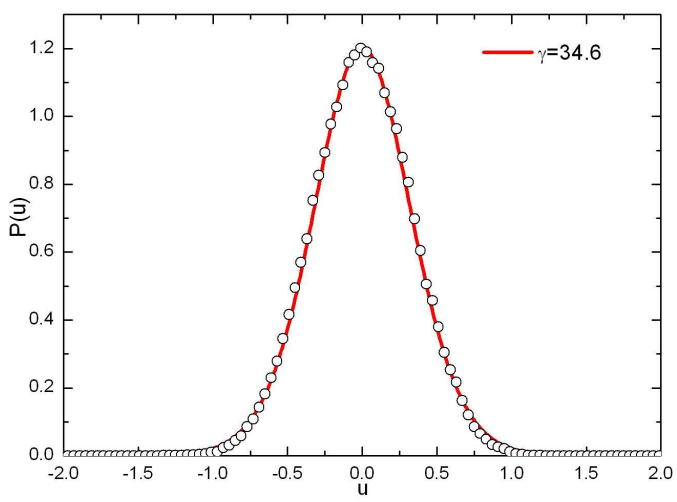

Fig. 7. The numerical distribution $P(u)$ of the real part of the $K$ matrix for the mean absorption parameter: $\bar{\gamma}=34.6$ (empty circles). The corresponding theoretical distribution $P(v)$ is marked by the solid line. 
with the exact theoretical predictions of Savin et al. [39] is observed. Only small deviations of $P(R)$ are seen in the vicinity of $R=0.08$. There is also some discrepancy of the distribution $P(v)$ nearby its maximum and hardly seen excess in the vicinity of $v$ equal to 1.4.

\section{Experimental investigation of the irregular 3D microwave cavity}

The irregular 3D microwave cavity was made of polished aluminum type EN 5754, whose density is $2.67 \mathrm{~g} / \mathrm{cm}^{3}$. It consists of a rough half-circle element of maximum height $h=60 \mathrm{~mm}$ closed by two flat elements, side and upper. The third, bottom element is formed in shape of irregular convex to remove the translational symmetry of the cavity (see Fig. 8 for dimensions and shape details). The rough element is described on the cross-section plane by the radius function $R(\theta)=R_{0}+\sum_{m=2}^{M} a_{m} \sin \left(m \theta+\Phi_{m}\right)$, where the mean radius $R_{0}=10.0 \mathrm{~cm}, M=20, a_{m}$ and $\Phi_{m}$ are uniformly distributed on $[0.084,0.091] \mathrm{cm}$ and $[0,2 \pi]$, respectively, and $0 \leq \theta<\pi$. It is a modification of the cavity with a flat bottom element, which we used in our previous experiments [33, 34].
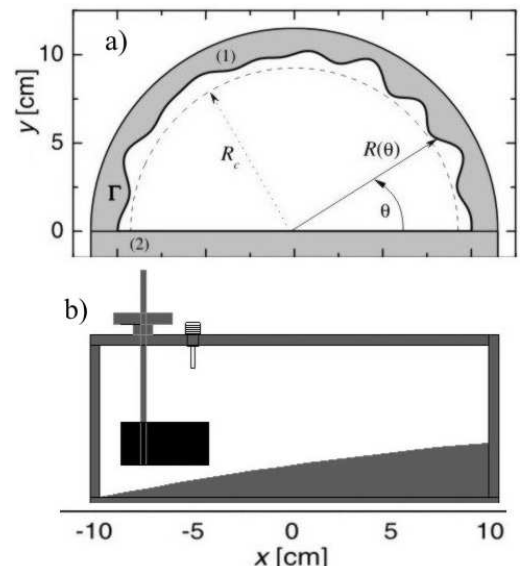

c)

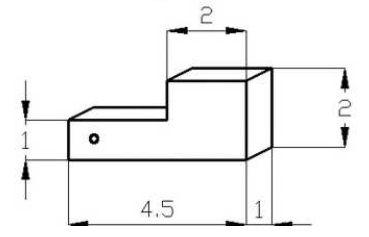

Fig. 8. The shape and the dimensions in $\mathrm{cm}$ of the $3 \mathrm{D}$ rough cavity and the foam absorber. (a) The sketch of the cavity in the $x y$ plane. (b) The sketch of the cavity in the $x z$ plane. The positions of the antenna and the axle with the absorber is shown. All dimensions are in the scale. (c) The foam absorber seen from the top of the cavity. The axle position is marked by a circle.

Number of eigenfrequencies of the empty, weakly absorbing cavity can be roughly evaluated from the BalianBloch formula [61]:

$$
N(k) \simeq \frac{1}{3 \pi^{2}} V k^{3},
$$

where $k$ is the wave vector, $V=(7.337 \pm 0.012) \times 10^{-4} \mathrm{~m}^{3}$ is the volume of the cavity.

The foam L-shape microwave absorber of the volume about $0.00001 \mathrm{~m}^{3}$ (type RFRET, \# 4009, product of Laird Technology) was inserted inside the cavity in order to increase its absorption (see Fig. 8). The absorber was mounted on the metallic axle. This construction allowed for turning the absorber around the axle as well as for changing its position along $z$ direction, parallel to the height of the cavity.

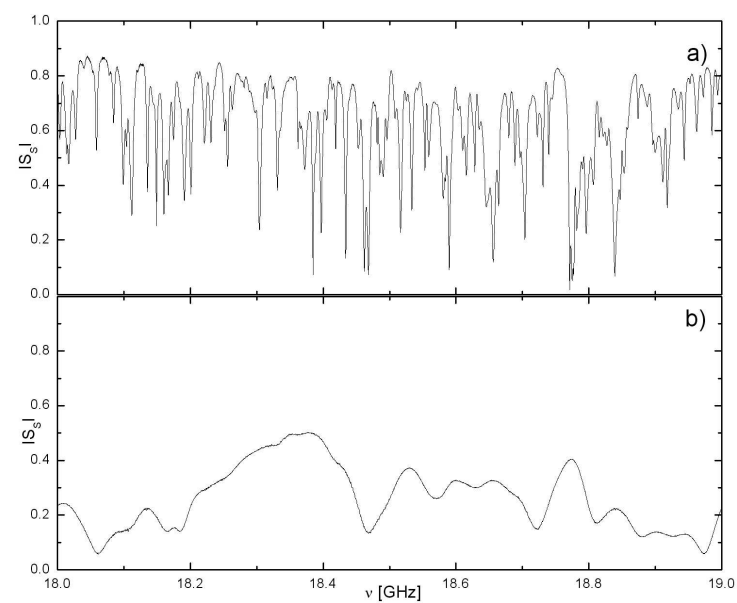

Fig. 9. (a) The spectrum of the empty cavity, without absorber, in the frequency range 18-19 GHz. (b) The spectrum of the cavity with the absorber in the same frequency range.

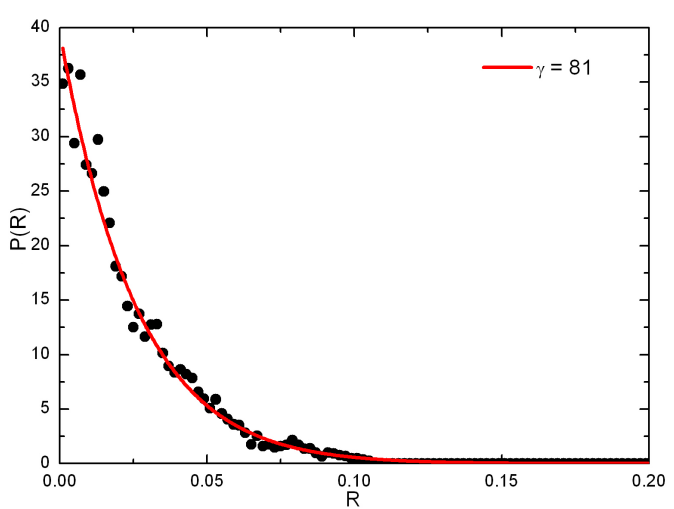

Fig. 10. The experimental distribution $P(R)$ of the reflection coefficient $R$ for the 3D microwave rough cavity for $\bar{\gamma}=81.0$ (solid circles). The corresponding theoretical distribution $P(R)$ is marked by the solid line.

The measurements of the scattering matrix $S_{\mathrm{s}}$ for the $3 \mathrm{D}$ cavity were done in the frequency range $18-19 \mathrm{GHz}$, where, on the basis of the formula (14), the variation of the mean eigenfrequency spacing $\Delta_{f}$ is reduced to $\delta \Delta_{f} / \Delta_{f} \approx 0.12$. The moduli of the scattering ma- 
trix $\left|S_{\mathrm{S}}\right|$ of the cavity without and with the absorber are shown in Fig. 9. The distribution $P(R)$ shown in Fig. 10 is the result of the averaging measurements for different positions of the absorber inside the cavity which resulted in $\bar{\gamma}=81.0$. The position of the absorber was changed by turning the axle around in 28 equal steps, each having the value $\pi / 36$ radians. The absorber was placed nearby the bottom of the cavity and its position along the $z$ direction was not changed during the measurements. The agreement with the exact theoretical results predicted for quantum systems is astonishingly good taking into account that the $3 \mathrm{D}$ cavity has no formal analog in quantum 3D systems. However, in contrary to the previous results, the distributions of the imaginary and the real parts $P(v)$ and $P(u)$ of the $K$ matrix (not shown in the paper) obtained for the same value of the absorption parameter $\bar{\gamma}=81.0$ showed significant deviations from the theoretical predictions. Finally, it should be stressed out that the obtained results are so interesting from the point of view of $3 \mathrm{D}$ microwave cavity - 3D quantum system analogy that they should trigger further investigations in this field. It appeared for example that absorption of the $3 \mathrm{D}$ cavity was not only dependent on the size and the position of the absorber but also on the position of the antenna.

\section{Summary}

In summary, we measured and calculated numerically the distribution of the reflection coefficient $P(R)$ and the distributions of the imaginary $P(v)$ and the real $P(u)$ parts of the Wigner reaction $K$ matrix for irregular fully connected hexagon networks and quantum graphs in the presence of strong absorption. The application of attenuators allowed for effective change of absorption in the networks. In the numerical calculations absorption in an attenuator was modelled by an optical potential. We showed that in the case of preserved time reversal symmetry $(\beta=1)$ the experimental and numerical results for $P(R), P(v)$ and $P(u)$ are in good agreement with the theoretical predictions.

In the case of the $3 \mathrm{D}$ microwave rough cavity we measured the distribution of the reflection coefficient $P(R)$ in the presence of strong absorption. In spite of the fact that the cavity does not possess a formal analog with a 3D quantum system the experimental distribution of the reflection coefficient $P(R)$ appeared to be in a reasonable agreement with the theoretical one predicted for quantum systems.

\section{Acknowledgments}

This work was partially supported by the Ministry of Science and Higher Education grant No. N202 099 $31 / 0746$.

\section{References}

[1] L. Pauling, J. Chem. Phys. 4, 673 (1936).

[2] C. Flesia, R. Johnston, H. Kunz, Europhys. Lett. 3, 497 (1987).

[3] R. Mitra, S.W. Lee, Analytical Techniques in the Theory of Guided Waves, Macmillan, New York 1971.

[4] Y. Imry, Introduction to Mesoscopic Systems, Oxford, New York 1996.

[5] D. Kowal, U. Sivan, O. Entin-Wohlman, Y. Imry, Phys. Rev. B 42, 9009 (1990).

[6] E.L. Ivchenko, A.A. Kiselev, JETP Lett. 67, 43 (1998).

[7] J.A. Sanchez-Gil, V. Freilikher, I. Yurkevich, A.A. Maradudin, Phys. Rev. Lett. 80, 948 (1998).

[8] Y. Avishai, J.M. Luck, Phys. Rev. B 45, 1074 (1992).

[9] T. Nakayama, K. Yakubo, R.L. Orbach, Rev. Mod. Phys. 66, 381 (1994).

[10] T. Kottos, Acta Phys. Pol. A 109, 7 (2006).

[11] T. Kottos, U. Smilansky, Phys. Rev. Lett. 79, 4794 (1997).

[12] F. Barra, P. Gaspard, J. Statist. Phys. 101, 283 (2000).

[13] R. Blümel, Yu. Dabaghian, R.V. Jensen, Phys. Rev. Lett. 88, 044101 (2002).

[14] G. Tanner, J. Phys. A 33, 3567 (2000).

[15] P. Pakoński, K. Życzkowski, M. Kuś, J. Phys. A 34 , 9303 (2001)

[16] O. Hul, S. Bauch, P. Pakoński, N. Savytskyy, K. Życzkowski, L. Sirko, Phys. Rev. E 69, 056205 (2004).

[17] O. Hul, O. Tymoshchuk, Sz. Bauch, P.M. Koch, L. Sirko, J. Phys. A 38, 10489 (2005).

[18] O. Hul, P. Seba, L. Sirko, Phys. Scr. T 135, 014048 (2009).

[19] M. Ławniczak, S. Bauch, O. Hul, L. Sirko, Phys. Scr. T 135, 014050 (2009).

[20] O. Hul, P. Seba, L. Sirko, Phys. Rev. E 79, 066204 (2009).

[21] H. Schanz, T. Kottos, Phys. Rev. Lett. 90, 234101 (2003).

[22] L. Kaplan, Phys. Rev. E 64, 036225 (2001).

[23] T. Kottos, H. Schanz, Physica E 9, 523 (2003).

[24] T. Kottos, U. Smilansky, J. Phys. A 36, 3501 (2003).

[25] M. Ławniczak, O. Hul, S. Bauch, P. Seba, L. Sirko, Phys. Rev. E 77, 056210 (2008).

[26] G. Akguc, L.E. Reichl, Phys. Rev. E 64, 056221 (2001).

[27] Y.V. Fyodorov, D.V. Savin, JETP Lett. $\mathbf{8 0}, 725$ (2004).

[28] O. Hul, S. Bauch, M. Ławniczak, L. Sirko, Acta Phys. Pol. A 112, 655 (2007).

[29] S. Deus, P.M. Koch, L. Sirko, Phys. Rev. E 52, 1146 (1995).

[30] H. Alt, C. Dembowski, H.-D. Gräf, R. Hofferbert, H. Rehfeld, A. Richter, R. Schuhmann, T. Weinland, Phys. Rev. Lett. 79, 1026 (1997).

[31] U. Dörr, H.-J. Stöckmann, M. Barth, U. Kuhl, Phys. Rev. Lett. 80, 1030 (1998). 
[32] C. Dembowski, B. Dietz, H.-D. Gräf, A. Heine, T. Papenbrock, A. Richter, C. Richter, Phys. Rev. Lett. 89, 064101 (2002).

[33] O. Tymoshchuk, N. Savytskyy, O. Hul, Sz. Bauch, L. Sirko, Phys. Rev. E 75, 037202 (2007)

[34] N. Savytskyy, O. Tymoshchuk, O. Hul, Sz. Bauch, L. Sirko, Phys. Lett. A 372, 1851 (2008).

[35] H. Primack, U. Smilansky, Phys. Rev. Lett. 74, 4831 (1995).

[36] T. Prosen, Phys. Lett. A 233, 323 (1997).

[37] L.R. Arnaut, Phys. Rev. E 73, 036604 (2006).

[38] M.L. Mehta, Random Matrices, Academic Press, New York 1991

[39] D.V. Savin, H.-J. Sommers, Y.V. Fyodorov, JETP Lett. 82, 544 (2005).

[40] S. Hemmady, X. Zheng, T.M. Antonsen Jr., E. Ott, S.M. Anlage, Acta Phys. Pol. A 109, 65 (2006)

[41] S. Hemmady, X. Zheng, E. Ott, T.M. Antonsen, S.M. Anlage, Phys. Rev. Lett. 94, 014102 (2005).

[42] G. López, P.A. Mello, T.H. Seligman, Z. Phys. A 302 , 351 (1981).

[43] E. Doron, U. Smilansky, Nucl. Phys. A 545, 455 (1992).

[44] P.W. Brouwer, Phys. Rev. B 51, 16878 (1995).

[45] D.V. Savin, Y.V. Fyodorov, H.-J. Sommers, Phys. Rev. E 63, 035202 (2001).

[46] U. Kuhl, M. Martinez-Mares, R.A. Méndez-Sánchez, H.-J. Stöckmann, Phys. Rev. Lett. 94, 144101 (2005).

[47] E. Kogan, P.A. Mello, H. Liqun, Phys. Rev. E 61, R17 (2000).
[48] R.A. Méndez-Sánchez, U. Kuhl, M. Barth, C.V. Lewenkopf, H.-J. Stöckmann, Phys. Rev. Lett. 91, 174102 (2003).

[49] C.W.J. Beenakker, P.W. Brouwer, Physica E 9, 463 (2001).

[50] Y. Hlushchuk, L. Sirko, U. Kuhl, M. Barth, H.-J. Stöckmann, Phys. Rev. E 63, 046208 (2001).

[51] L. Sirko, P.M. Koch, R. Blümel, Phys. Rev. Lett. 78, 2940 (1997)

[52] Y. Hlushchuk, A. Kohler, S. Bauch, L. Sirko, R. Blümel, M. Barth, H.-J. Stöckmann, Phys. Rev. E 61, 366 (2000).

[53] N. Savytskyy, A. Kohler, S. Bauch, R. Blümel, L. Sirko, Phys. Rev. E 64, 036211 (2001).

[54] R. Blümel, P.M. Koch, L. Sirko, Foundations of Physics 31, 269 (2001).

[55] L. Sirko, A. Haffmans, M.R.W. Bellermann, P.M. Koch, Europhys. Lett. 33, 181 (1996)

[56] L. Sirko, S.A. Zelazny, P.M. Koch, Phys. Rev. Lett. 87, 043002 (2001).

[57] L. Sirko, P.M. Koch, Phys. Rev. Lett. 89, 274101 (2002).

[58] Microwave Cables and Assemblies General Catalogue, Vol. 51, Catalogue Huber and Suhner, 2007.

[59] P. Exner, Ann. Inst. H. Poincaré: Phys. Théor. 66, 359 (1997).

[60] G. Goubau, Electromagnetic Waveguides and Cavities, Pergamon Press, Oxford 1961.

[61] R. Balian, C. Bloch, Ann. Phys. (N.Y.) 84, 559 (1974) 